\title{
The Invasion and Reproductive Toxicity of QDs-Transierrin Bioconjugates on Preantral Follicle in vitro
}

\author{
Gaixia Xu' ${ }^{1,2}$, Suxia Lin², Wing-Cheung Law ${ }^{3}$, Indrajit Roy ${ }^{3}$, Xiaotan Lin², Shujiang Mei ${ }^{4}$, Hanwu Ma4, Siping \\ Chen², Hanben Niu1 ${ }^{1}$ Xiaomei Wang ${ }^{2 \varpi}$
}

1. College of Optoelectronic Engineering, Key Laboratory of Optoelectronics Devices and Systems of Ministry of Education/Guangdong Province, Shenzhen University, Shenzhen 518060, P. R. China

2. School of Medicine, Shenzhen Key laboratory of Biomedical Engineering, Shenzhen University, Shenzhen 518060, P. R. China

3. Institute for Lasers, Photonics and Biophotonics and Department of Electrical Engineering, The State University of New York at Buffalo, Buffalo, New York 14260-4200, U.S

4. The Center of Disease Prevent and Control in Shenzhen, Shenzhen 518020, P. R. China

$\triangle$ Corresponding author: E-mail: xugaixia@szu.edu.cn; xmwang@szu.edu.cn, Fax: 86-755-2653-8556, Tel: 86-755-2653-8580.

(C) Ivyspring International Publisher. This is an open-access article distributed under the terms of the Creative Commons License (http://creativecommons.org/ licenses/by-nc-nd/3.0/). Reproduction is permitted for personal, noncommercial use, provided that the article is in whole, unmodified, and properly cited.

Received: 2012.02.26; Accepted: 2012.04.30; Published: 2012.08.01

\begin{abstract}
The toxicity of QD has been extensively studied over the past decade. However, the potential toxicity of QDs impedes its use for clinical research. In this work, we established a preantral follicle in vitro culture system to investigate the effects of QD-Transferrin (QDs-Tf) bioconjugates on follicle development and oocyte maturation. The preantral follicles were cultured and exposed to CdTe/ZnTe QDs-Tf bioconjugates with various concentrations and the reproductive toxicity was assessed at different time points post-treatment. The invasion of QDs-Tf for oocytes was verified by laser scanning confocal microscope. Steroid production was evaluated by immunoassay. C-band Giemsa staining was performed to observe the chromosome abnormality of oocytes. The results showed that the QDs-Tf bioconjugates could permeate into granulosa cells and theca cells, but not into oocyte. There are no obvious changes of oocyte diameter, the mucification of cumulus-oocyte-complexes and the occurrence of aneulpoidy as compared with the control group. However, delay in the antrum formation and decrease in the ratio of oocytes with first polar body were observed in QDs-Tf-treated groups. The matured oocytes with first polar body decreased significantly by $\sim 16 \%$ (from $79.6 \pm 10 \%$ to $63 \pm 2.9 \%$ ) when the concentration of QDs-Tf bioconjugates exceeded $2.89 \mathrm{nmol} \mathrm{L}^{-1}(\mathrm{P}<0.05)$. Our results implied that the CdTe/ZnTe QDs-Tf bioconjugates were reproductive toxic for follicle development, and thus also revealed that this in vitro culture system of preantral follicle is a highly sensitive tool for study on the reproductive toxicity of nanoparticles.
\end{abstract}

Key words: preantral follicle; invasion; reproductive toxicity; in vitro culture system; QDs-transferrin bioconjugate.

\section{INTRODUCTION}

Over the past decade, luminescent semiconductor, also known as quantum dots (QDs), have been applied in biomedical imaging and drug delivery be- cause of their fascinating optical properties ${ }^{[1,2]}$. QDs conjugated with various kind of biomolecules (protein, antibody, peptide, DNA, etc.) have been used for 
cell labeling, single molecule tracking, diagnostics and targeted therapy [3-5]. Despite the wide application of QDs, a great deal of safety issue has been raised about the potential hazard of these nanomaterials to human and the environment [6,7]. Toxicity of QD has been extensively studied using in vitro and in vivo models ${ }^{[8,9]}$. Cell proliferation assay such as [3-(4, 5-dimethylthiazol-2-yl)-5-(3-carboxymethoxyphenyl)2-(4-sulfophenyl)-2H- tetrazolium (MTS) was widely used for the cytotoxicity assessment of nanomaterial. However, the result could be inconclusive as MTS assay can only provide general toxicity information. For more specific application, the evaluations of neurotoxicity, immunotoxicity, reproductive toxicity and genotoxicity may be necessary [10]. Generally, the evaluation of the female reproductive dysfunction by in vivo studies was carried out on the basis of parameters related to fertility outcome, which was time-consuming and unable to elucidate the mechanism of ovary damage [11], and only few reproductive toxicity studies were reported [12]. Fortunately, Sun and Cortvrindt improved the mouse follicle culture system that allowed the growth and development of intact early preantral follicles up to the ovulatory stage [11,13], which provided an excellent model for in vitro evaluation of ovarian functions $[14,15]$. They demonstrated that the preantral follicle in vitro growth for reproductive toxicity testing was reliable and had 10 times more sensitivity than the conventional in vivo study ${ }^{[13]}$.

In our previous work, we have established the preantral follicles (PFs) in vitro culture system and applied the three-dimension (3D) tomography to visualize the invasion of lysine coated QDs for oocytes [16]. The results showed that the QDs dramatically decreased the maturity rate of oocytes. It is worth mentioning that the hydrodynamic diameter of lysine coated QD is $\sim 20 \mathrm{~nm}$. In this work, ultrasmall $\mathrm{CdTe} / \mathrm{ZnTe}$ QDs conjugated with targeting molecule, transferrin (Tf), were prepared and served as a nanovector. QDs are generally utilized in drug delivery and targeted imaging by conjugating with biomolecules, such as transferrin and folic acid, whose receptors are overexpressed on the specific cellular membrane $[17,18]$. The study on the reproductive toxicity of QDs bioconjugates, not QDs alone, has much significance for clinic trial. We hypothesized that the small hydrodynamic diameter and the bioconjugation of QD can facilitate the penetration and induce more impact on oocytes [19]. After oocytes in droplets were exposed to QDs-Tf bioconjugates, the reproductive parameters, such as (i) preantral follicles growth/development, (ii) oocyte maturation and (iii) oocyte chromosomal aneuploidy, were analyzed.

\section{MATERIALS AND METHODS}

\section{Chemicals}

L-cysteine (99.5\%), cadmium perchlorate $\mathrm{Cd}\left(\mathrm{ClO}_{4}\right)_{2}$, zinc perchlorate $\left(\mathrm{Zn}\left(\mathrm{ClO}_{4}\right)_{2}\right)$, tellurium $(\mathrm{Te})$ powder $(99.8 \%, 200$ mesh), sodium borohydride $\left(\mathrm{NaBH}_{4}\right), \mathrm{N}$ - (3-Dimethylaminopropyl) -N- ethylcarbodiimide hydrochloride (EDC) were purchased from Aldrich. All the above chemicals were used as received to synthesized the L-cystein coated CdTe/ZnTe Core/Shell QDs .

Pregnant mare serum gonadotrophin (PMSG), human chorionic gonadotrophin (HCG), epidermal growth factor (EGF), insulin $\left(5 \mathrm{mg} \mathrm{mL}^{-1}\right)$ - transferrin (5 mg mL-1) - selenium (5 $\mathrm{ng} \mathrm{mL}^{-1}$ ) (ITS), luteinizing hormone (LH), N-2-Hydroxyethylpiperazine-N'-2ethanesulfonic acid (HEPES), follicle stimulating hormone (FSH), culture medium a-MEM (enriched with $200 \mathrm{mM}$ glutamine, $10 \%$ fetal bovine serum, ITS, $100 \mathrm{mIU} \cdot \mathrm{L}^{-1} \mathrm{FSH}$ and $\left.10 \mathrm{mIU} \cdot \mathrm{L}^{-1} \mathrm{LH}\right)$, isolation medium M2(containing $200 \mathrm{IU} \cdot \mathrm{mL}^{-1}$ penicillin+200 IU $\mathrm{mL}^{-1}$ streptomycin $+20 \mathrm{mmol} \cdot \mathrm{L}^{-1}$ HEPES, supplemented with 5\% FBS), stimulation medium M16(containing $200 \mathrm{IU} \cdot \mathrm{mL}^{-1} \mathrm{HCG}$ and $100 \mathrm{ng} \mathrm{mL}^{-1}$ EGF), mineral oil, penicillin, streptomycin and hyaluronidase were purchased from Sigma. Fetal bovine serum (FBS) was purchased from Gibco.

\section{Synthesis of cystein coated CdTe/ZnTe Core/Shell QDs}

The synthesis method of CdTe/ZnTe Core/Shell QDs was adapted from that presented by Law et al[20]. Briefly, $127 \mathrm{mg}$ of tellurium powder and $100 \mathrm{mg}$ of sodium borohydride were mixed with $5 \mathrm{~mL}$ of nitrogen-saturated water. The mixture was sealed and stirred for 1-2 hours until it became light-purple color. This solution is referred as the Te precursor. The next step, $1 \mathrm{mmol}$ of cadmium perchlorate, $2 \mathrm{mmol}$ of zinc perchlorate, $4 \mathrm{mmol}$ of cysteine, and $125 \mathrm{~mL}$ of nitrogen-saturated water were loaded into a $250 \mathrm{~mL}$ three-necked flask under stirring. The $\mathrm{pH}$ was adjusted 10-11 by adding dropwise sodium hydroxide solution until a clear solution was formed. The flask was sealed and subsequently $1.5 \mathrm{~mL}$ of Te precursor was injected into the mixture under nitrogen atmosphere. The reaction mixture was slowly heated to 100 ${ }^{\circ} \mathrm{C}$. The QDs were extracted when red emission was observed under the irradiation of hand-held UV lamp. The QDs were separated from the surfactant solution by the addition of ethanol and centrifugation. It has been demonstrated that the QDs had good biological stability and optical stability [21]. In this work, the QDs emitted red fluorescence with peak at $635 \mathrm{~nm}$ when excited. The zeta potential and size of cysteine coated 
QD is $\sim 3 \mathrm{mV}$ and $\sim 4 \mathrm{~nm}$ respectively.

\section{Conjugation of CdTe/ZnTe QDs with trans- ferrin}

From the stock solution, $0.1 \mathrm{~mL}$ QDs solution was mixed with $0.2 \mathrm{~mL}$ of $2.5 \mathrm{mM}$ EDC solution and settled for $5 \mathrm{~min}$. Next, $0.1 \mathrm{~mL}$ of Tf $\left(2 \mathrm{mg} \mathrm{mL}^{-1}\right)$ was added into this mixture and incubated at room temperature for 2 hours to allow the protein to covalently bond to the QDs.

\section{Animals}

Female Kunming mice provided by Guangdong Laboratory Animal Center (Guangzhou) were housed in groups in a temperature and light-controlled room at $23-25^{\circ} \mathrm{C}$, on a 12 -hour light: 12 -hour dark light cycle and fed with pellet food and water as desired. Animal handling was in accordance with Shenzhen University standard animal husbandry practice and regulation.

\section{Preantral follicles collection}

Female mice with 12-day-old were sacrificed by cervical dislocation. The ovaries were aseptically removed from the animals and placed in the prewarmed isolation medium M2. Only preantral follicles with a diameter between 100-130 $\mu$ m containing an immature oocyte, which centrally located within the follicle and an intact basal membrane surrounded by some theca cells, were collected under stereomicroscope for follicle in vitro culture [22]. About 50-60 qualified follicles were harvested each ovary. All selected follicles were pooled and cultured according to the protocol adapted from Sun et al [13].

\section{Follicles culture and development}

After washing thrice in isolation medium M2 and washing twice in culture medium $\alpha$-MEM, the preantral follicles were cultured individually in $10 \mu \mathrm{L}$ droplets of culture medium overlaid with mineral oil in $35 \mathrm{~mm}$ dishes (10 droplets/dish). After incubation in vitro for 48 hours, $10 \mu \mathrm{L}$ of culture medium was added to each droplet. Subsequent refreshments were conducted every day by removing and replacing 10 $\mu \mathrm{L} \alpha$-MEM. The morphology of follicles was observed and measured daily under inverted microscope and the ratio of survival follicles, antrum cavity formation and matured oocytes for both treated and control groups were calculated. On the eighth day, the follicles were replenished by stimulation medium M16 in the culture droplet. After the stimulation by HCG and EGF for 16 hours, the cumulus-oocyte-complexes (COCs) were released. Then, according to their meiotic stages, the oocytes were classified as germinal vesicle $(\mathrm{GV})$ oocytes, germinal vesicle breakdown (GVBD) oocytes and oocytes with first polar body (PB) using a stereomicroscope. After that, the oocyte chromosomes were stained with DAPI [23]. GVBD and PB oocytes were spread for chromosomal analysis.

\section{Steroid production analysis}

The follicle culture media were collected and diluted for steroid production analysis by a commercially available Chemiluminescent Immunoassay (Beckman Coulter UniCel DXI 800, USA). The production of $17 \beta$-estradiol was monitored on the $4^{\text {th }}, 6^{\text {th }}$ and $8^{\text {th }}$ days of follicles in vitro culture, and the progesterone concentration was quantified on the $9^{\text {th }}$ day.

\section{Follicles in vitro exposure to QDs-Tf}

Once the theca cells attached onto the dish bottom, the preantral follicles were randomly divided into four QDs-Tf-treated groups and one control group. The QDs-Tf-treated groups were added with QDs-Tf bioconjugates, diluted by a-MEM at escalating concentrations of $0.0289 \mathrm{nmol} \cdot \mathrm{L}^{-1}, 0.289 \mathrm{nmol} \cdot \mathrm{L}^{-1}$, $2.89 \mathrm{nmol} \mathrm{L}^{-1}$ and $28.9 \mathrm{nmol} \cdot \mathrm{L}^{-1}$, respectively.

\section{Chromosomes spreading and C-band Giemsa staining}

Normally, the GVBD oocytes had two sets of chromosomes which were in anaphase I, and PB oocytes had two sets of chromosomes and visibly underwent cytokinesis. The GVBD and PB oocytes were selected and spread according to the standard methods [24]. Briefly, GVBD and PB Oocytes were treated with $0.75 \%$ hypotonic sodium citrate at room temperature for 20 mins. 10 15 oocytes had been immersed in fixing buffer (acetic acid: methanol=1:3) for 5 mins, then, $3 \sim 5$ fixed oocytes were immediately transferred onto an ice-cold slide, and finally, the slide was air dried. Then, all slides were stained by C-band Giemsa.

\section{Fluorescence microscopic imaging}

The fluorescence images were taken under an inverted fluorescence microscope (Olympus, IX71, Japan). The z-scanning images were taken by a laser scanning confocal microscope (TCS SP2, Leica, Germany) to confirm the QDs-Tf location in follicle with an excitation wavelength at $488 \mathrm{~nm}$. The z-stack reflection and fluorescence images of a single follicle were acquired by means of optical sectioned imaging, and the z-positions of these images were selected to fit with the Nyquist sampling theorem [25]. Generally, we set the z-scanning parameters (scanning range and step length) according to the follicle size. All the reflection and fluorescence images from the same 
z-positions were overlapped together to render the reconstructed images. The gray level of each pixel of the reconstructed fluorescence image was summed, as a quantificational parameter in describing the quantity of invasion.

\section{Statistical analysis}

The impacts of QDs-Tf for follicle development and chromosomal aneuploidy were analyzed by Pearson's Chi-square test. The effects of QDs-Tf for steroid production and oocyte maturity were analyzed using one-way ANOVA test. A P-value of less than 0.05 was considered to be statistically significant. The measurements were repeated at least 4 times and the data was expressed as the mean \pm standard deviation (SD). All statistical calculations were done with the SPSS software package.

\section{RESULTS AND DISCUSSION}

\section{In vitro development of preantral follicles}

To establish the in vitro model for the development of preantral follicles, we dissected the ovary by mechanical means from female mice (Figure 1A), and harvested the qualified preantral follicles with granulosa cells and theca cells on the basal membrane (Figure 1B).

Then, the preantral follicles were culture and observed under microscope. The morphological development of follicles and in vitro ovulation at different stages were shown in Figure 2. On the isolation day of follicle in vitro culture, a precise class of preantral follicles (Figure 2A) with similar diameter $(100-130 \mu \mathrm{m})$ containing an immature oocyte, which centrally located within the follicle and an intact basal membrane surrounded by $40 \sim 80$ theca cells, were selected for follicle culture. On the second day of culture, theca cells attached to the dish bottom (Figure
2B). The granulosa cells proliferated and grew rapidly and the diameter of follicles increased dramatically, as shown in Table 1. On the sixth day of culture, with the proliferation and differentiation of the granulosa cells, a fluid-filled antrum-like cavity and the COCs were formed (Figure 2C). After being stimulated by HCG and EGF for 16 hours, COCs were released from the follicles (Figure 2D). After removal of the cumulus cells, the mature metaphase II oocyte with first polar body and the zona pallucida were harvested as shown in Figure 2E. Two sets of chromosomes in PB oocyte were clearly resolved under an inverted fluorescence microscope (Figure 2F).

At the end of the culture, the rates of survival, antrum formation and mucification were statistically calculated $(n=128)$. The survival rate of follicle was $98.4 \%$, the antrum formation was $70.6 \%$ (shown in Table 2) and all follicles released mucified COCs, indicating that our preantral follicle in vitro culture system worked well and the follicle developed normally.

Table I. Growth of preantral follicle (mean $\pm S D)$.

\begin{tabular}{llllll}
\hline Day & $N$ & 0 & 2 & 4 & 6 \\
\hline $\begin{array}{l}\text { Diameter } \\
(\mu \mathrm{m})\end{array}$ & 160 & $140 \pm 10$ & $202 \pm 35$ & $310 \pm 42$ & $331 \pm 45$ \\
\hline
\end{tabular}

(N, number of preantral follicle)

Table 2. Development potential of preantral follicles in vitro.

\begin{tabular}{lccll}
\hline Group & $\mathrm{N}$ & Survival (\%) & $\begin{array}{l}\text { Antrum for- } \\
\text { mation (\%) }\end{array}$ & Mucification (\%) \\
\hline $\begin{array}{l}\text { In Vitro } \\
\text { Culture }\end{array}$ & 128 & $98.4(126 / 128)$ & $70.6(89 / 126)$ & $100(89 / 89)$ \\
\hline
\end{tabular}

(N, number of preantral follicle)
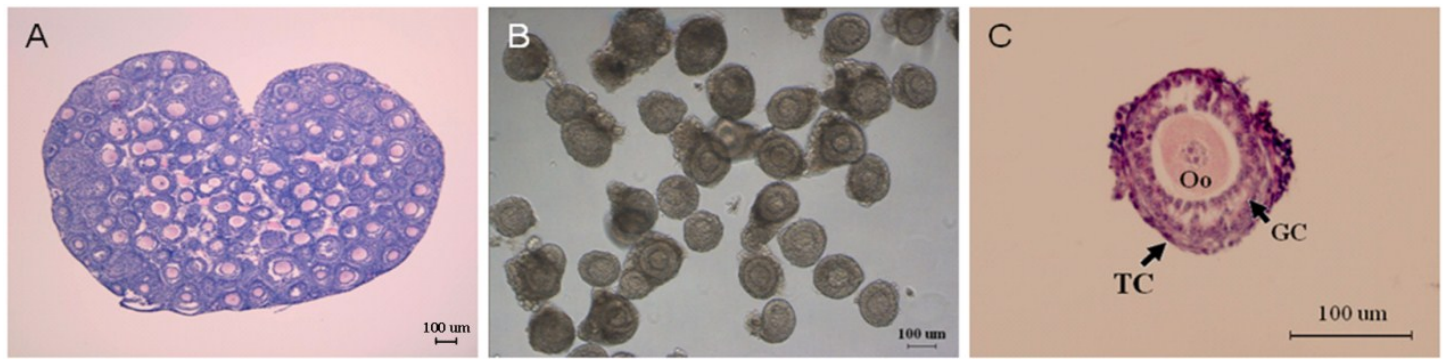

Figure I. Morphology of ovary and preantral follicles. (A) Mouse ovary, H\&E staining. (B) Preantral follicles dissected mechanically. (C) Fixed single preantral follicle, H\&E staining, Oo: oocyte; GC: granulosa cell; TC: theca cell. 


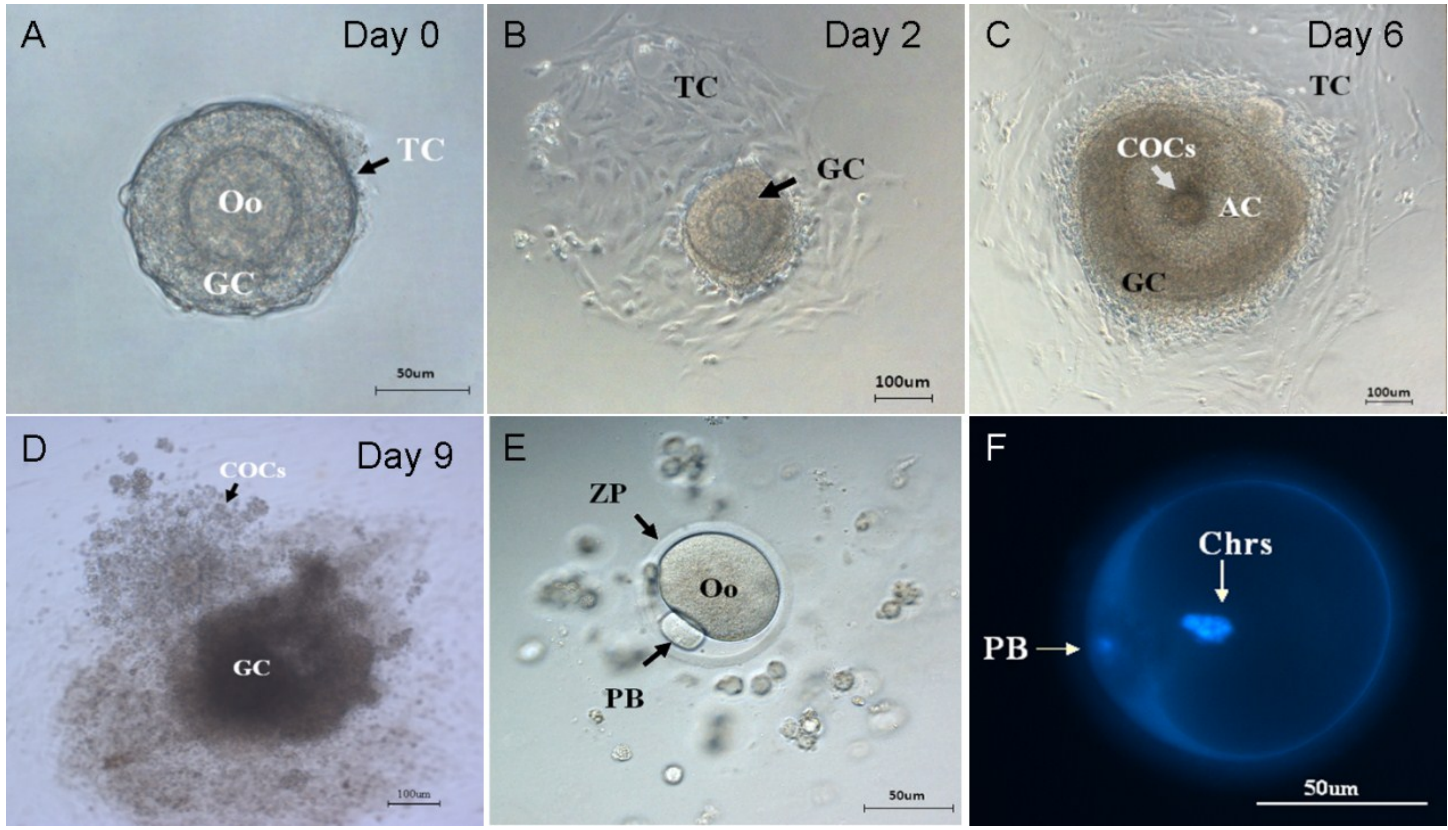

Figure 2. Stages of folliculogenesis and oocyte maturation. (A) On the isolation day, intact preantral follicle was transferred into dishes, surrounded by granulosa cells and theca cells. (B) On the $2^{\text {nd }}$ day, the theca cells attached to the bottom of culture dishes. (C) On the 6 th day, the granulosa cells proliferated and differentiated, the antrum and COCs were formed. (D) On the 9th day, COCs was released under the stimulation of HCG and EGF. (E) On the 9th day, the granulosa cells were removed and the single oocytes with first polar body was denuded. (F) On the 9th day, two sets of chromosomes of oocyte stained by DAPI. Oo:oocyte; GC: granulosa cell; TC: Theca cell; COCs: Cumulus-Oocyte-Complexes; AC: Antrum cavity; PB: polar body; ZP: Zona pellucida; Chrs: Chromosome.

\section{Distribution of QDs-Tf in preantral follicle in vitro}

In order to study the invasion of the nanoparticles, the distribution of QDs-Tf in preantral follicle was monitored using confocal microscopy. All selected preantral follicles $(100-130 \mu \mathrm{m})$ from different ovaries were pooled and randomly divided into four treated groups and one control group. Once the theca cells attached the dish bottom (Figure 3A), the QDs-Tf with various concentration $0.0289 \mathrm{nmol} \cdot \mathrm{L}^{-1} 、 0.289$ $\mathrm{nmol} \cdot \mathrm{L}^{-1} 、 2.89 \mathrm{nmol} \cdot \mathrm{L}^{-1}$ and $28.9 \mathrm{nmol} \cdot \mathrm{L}^{-1}$ were added into culture dishes. Then the samples were observed by fluorescence microscope after different durations of incubations, as shown in Figure 3. The results revealed that the fluorescence of QDs-Tfs (red dots) could be easily found near the outer wall (theca cells) and the inner wall (granulosa cell) of follicle membrane. However, we didn't found obvious red fluorescence from QDs-Tf inside oocytes. Such phenomenon implied that the nanoparticles were uptaken mostly by theca cells and granulosa cells which was served as the first protective barrier of follicle in vitro system to resist exogenous substances from entering the central oocyte. Furthermore, we found that the QDs-Tf accumulated in the cytoplasm of theca cells and granulosa cells, as indicated by the small red sparkles in Figure 3D. Other researchers have also observed similar results that the gold nanoparticles could enter the ovarian granulosa cells and accumulate in storage vacuole, lipid droplet and mitochondria [26].

In order to verify our speculation, the preantral follicles were exposed to QDs-Tf with various concentrations (shown in Figure 3E-3H). One can observe that the accumulation of QDs-Tf increased significantly in theca cells and granulosa cells when the dosage was increased. At the concentration of $28.9 \mathrm{nmol} \cdot \mathrm{L}^{-1}$, the whole oocyte seems surrounded by the red cloud of QDs-Tf bioconjugates (Figure 3H).

To obtain a better portrait of QDs-Tf penetration, an optical sectioning experiment by laser scanning confocal microscope was performed to provide z-scanning images. The z-stack reflection and fluorescence images of a single oocyte were acquired by means of optical sectioned imaging with step length of $0.25 \mu \mathrm{m}$ (Figure 4). Obviously, the oocyte periphery was clear, which indicated that the zona pellucida was intact. Most of the fluorescence signals were found in the cumulus granulosa cells, indicating that the QDs-Tfs were uptaken by cumulus granulosa cells but none of them can get into the oocytes within the QDs-Tf concentrations used in this work. According to the anatomic morphology of follicle, the mecha- 
nism of the invasion of QDs-Tf to follicle can be outlined as following: nanosized bioconjugates can be uptaken by granulosa cells and penetrate the first protective of follicle; then, the zona pellucida worked as the second protective barrier to block the bioconjugates from entering the oocyte.

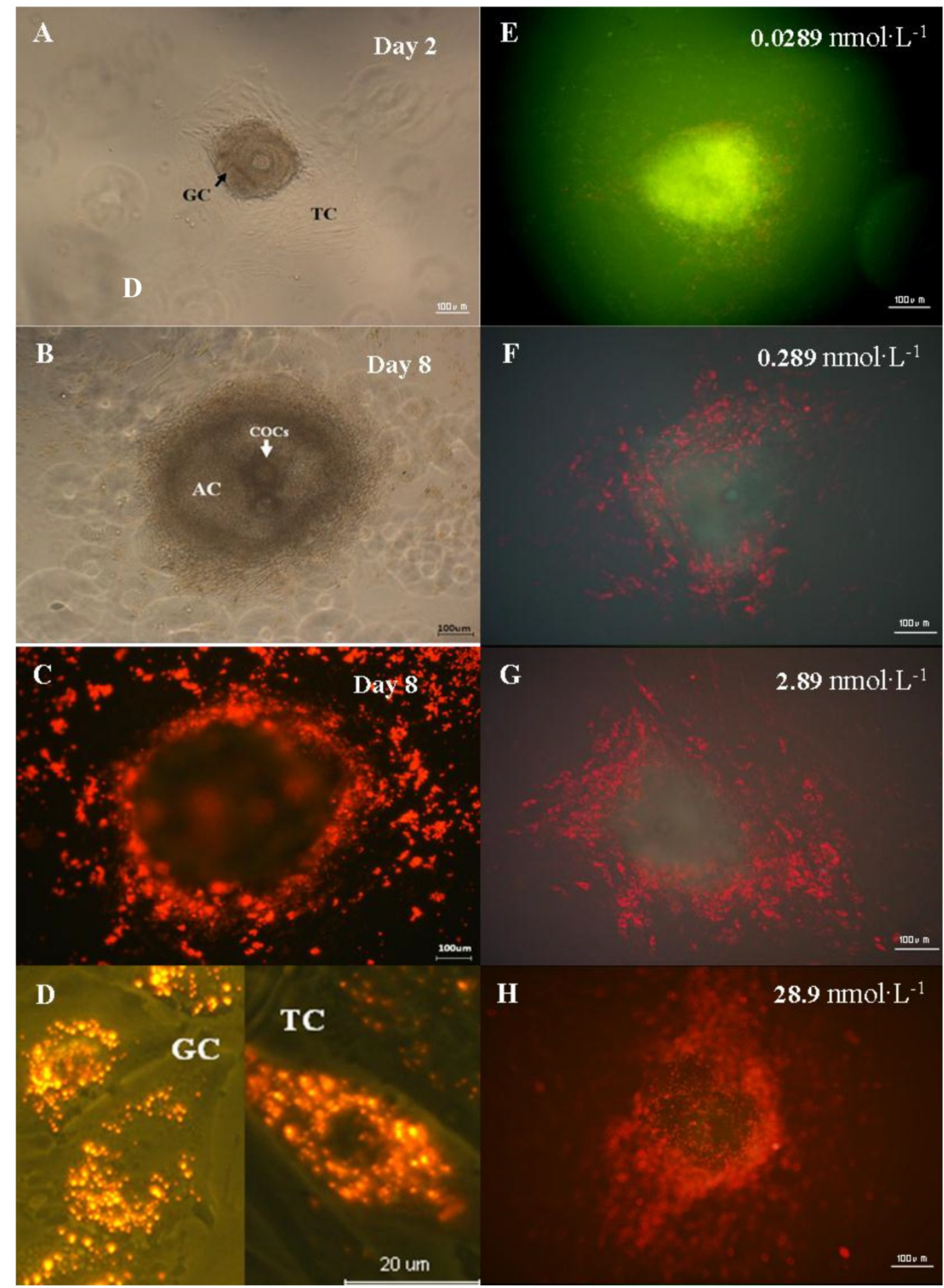

Figure 3. Distribution of QDs in the preantral follicles in vitro. (A) Preantral follicles cultured in vitro under phase-contrast microscope on the $2^{\text {nd }}$ day. (B) Follicles with antrum cavity cultured in vitro under phase-contrast microscope on the $8^{\text {th }}$ day. (C) Fluorescence microscopic image of follicles cultured in vitro on the $8^{\text {th }}$ day. (D) Magnified fluorescence microscopic image of theca cells and granulosa cells on the $8^{\text {th }}$ day. (E)-(H) Fluorescence microscopic image of follicle treated with QDs-Tf at different concentrations on the $8^{\text {th }}$ day. The green pseudo-color represented emission from autofluorescence of samples and the red ones from QDs. 

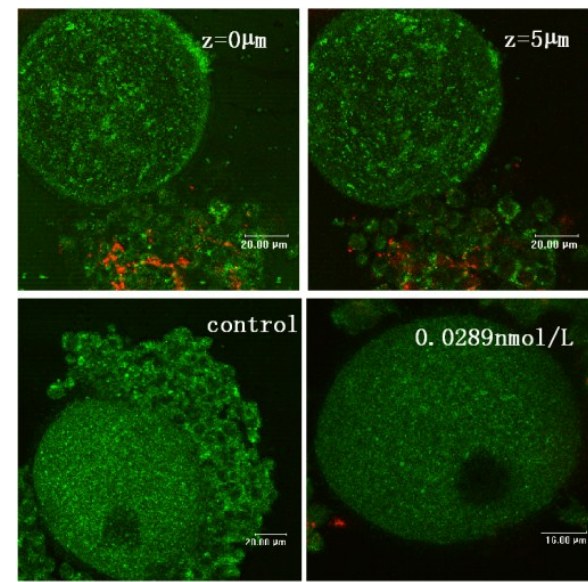

$0.0289 \mathrm{nmol} / \mathrm{L}$
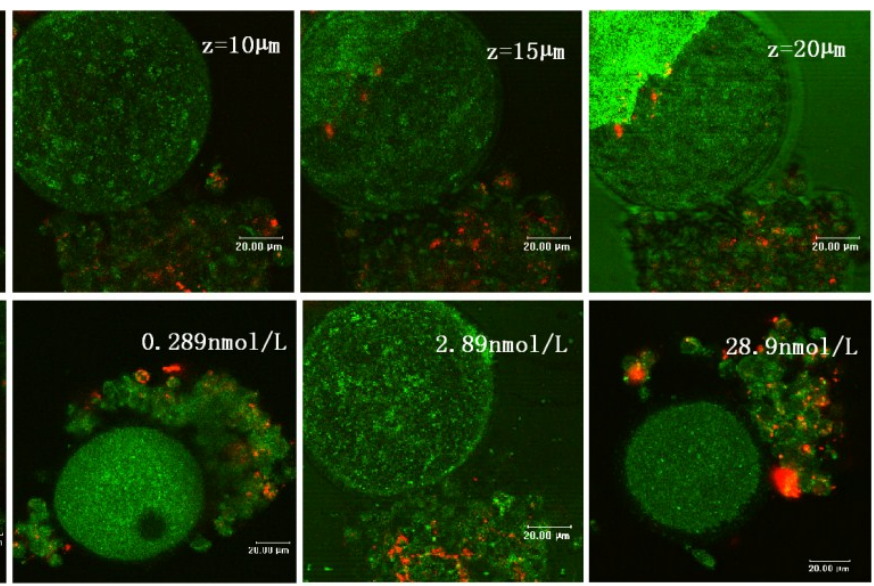

Figure 4. The confocal microscopic images of oocyte treated with QDs-Tf for 8 days. Upper panel: optical sectioning images of the oocyte treated with QDs-Tf $\left(2.89 \mathrm{nmol} \mathrm{L}^{-1}\right)$. Lower panel: the oocytes treated with QDs-Tf at different concentrations. Red pseudo-color represented fluorescence from QDs and green ones from reflecting signal.

\section{Effect of QDs-Tf on in vitro cultured follicles}

To investigate the induced toxicity of QDs to immature follicles, we compared the rates of survival follicles, antrum cavity formation and COCs mucification of treated groups with the control group. During the last period of in vitro culture, granulosa cells progressively differentiated into two subpopulations: steroid-producing mural granulosa cells and the cumulus cells tightly enclosing the oocyte (Figure 5B $\& 5 \mathrm{C})$. The survival follicles and antrum cavity formation were counted after the treatment of QDs-Tf for 8 days using stereomicroscope. Then, the follicles were treated with HCG and EGF to induce ovulation. Afterwards, the mucification of COCs, profiling with an expanded mass of cumulus granulosa cells, was observed and counted (Figure 5D). The results showed that the ratio of survival follicle and COCs mucification in treated groups had not significant differences with the control group $(\mathrm{P}>0.05)$. However, the rate of antrum cavities decreased from $91 \%$ to 70 $\%$ when the QDs-Tf concentration is higher than 2.89 $\mathrm{nmol} \cdot \mathrm{L}^{-1}(\mathrm{P}<0.05)$, as shown in Figure 5A. Considering the QDs-Tf accumulated in granulosa cells and theca cells (Figure 3D), we deduced that the high uptakes of QDs-Tf in theca cells and granulosa cells could potentially induce reproductive toxicity and consequently disturb the oocyte to form antrum cavity, which agreed with our previous work[16,27].

\section{Effect of QDs-Tf on steroid production}

Steroid hormone production is one of the most important parameters to evaluate the functions of theca cells and granulosa cells. During folliculogenesis, the theca cells respond to LH and synthesize androgens. Once the theca cells differentiate into an- drogen-producing cells, the granulosa cells start to produce estradiol[10].

Thus, in our work, the preantral follicle culture media were collected and the secretion of 17 $\beta$-estradiol was examined on the $4^{\text {th }}, 6^{\text {th }}$ and $8^{\text {th }}$ day, respectively (Figure 6). With the development of follicle, the production of $17 \beta$-estradiol increased rapidly in all groups. After in vitro culture for 4 days, the $17 \beta$-estradiol secretion in treated groups was progressively decreased from basal control level, depending upon the dosage of nanoparticles, which had no significant difference $(p>0.05)$. We deduced that the tranferrin proteins on the QDs-Tf at lower concentration were totally identified and the QDs-Tf bioconjugates were uptaken into the cells by transferrin receptors on the membrane of theca cell and granulosa cells. The accumulation of such nanoparticles inside cells disturbed the steroid biosythetic pathways and decreased the estradiol production [26]. However, it was interesting that the production of $17 \beta$-estradiol increased when treated with the QDs-Tf at highest concentration. We speculated that at highest concentration, higher uptake of QDs-Tf by granulosa cells altered the activity of the side chain cleavage enzyme which is located on the inner membrane of mitochondria and converts cholesterol to pregnenolone [19, 26]. Nevertheless, further experiments are necessary to understand the full mechanism of these phenomena.

The growth and development of follicles in culture are FSH-dependent and LH-responsive [28, 29]. To understand the relationship between the dosage of QDs-Tf and production of progesterone, preantral follicles treated with various concentration of QDs-Tf were stimulated to release COCs by HCG and EGF on the $8^{\text {th }}$ day of incubation. Then, the culture media were collected for evaluation of progesterone secre- 
tion. It was demonstrated that the production of progesterone had no significant difference between different dosage groups treated with QDs-Tf $(\mathrm{P}>0.05)$
(Figure 7), which suggested the QDs-Tf did not affect the production of progesterone.
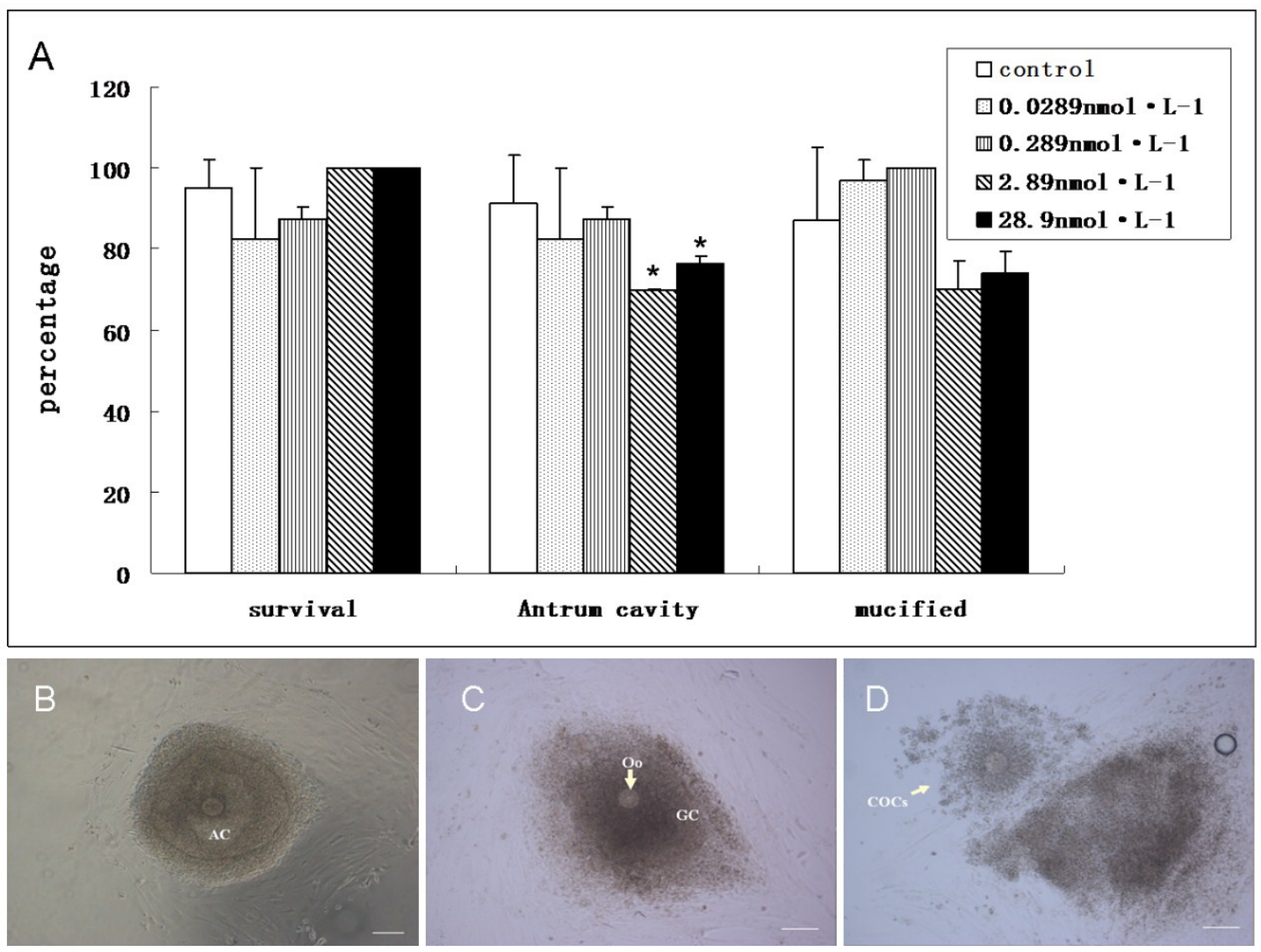

Figure 5. (A)Comparison about the ratio of survival follicle, antrum cavity formation and COCs mucification between control and treated groups. (mean $\pm \mathrm{SD},{ }^{*} \mathrm{P}<0.05$ vs control, Chi-Square test). (B) Follicle with antrum cavity. (C) Follicle without antrum cavity. (D) Mucified COCs. Scale bar: $100 \mu \mathrm{m}$.

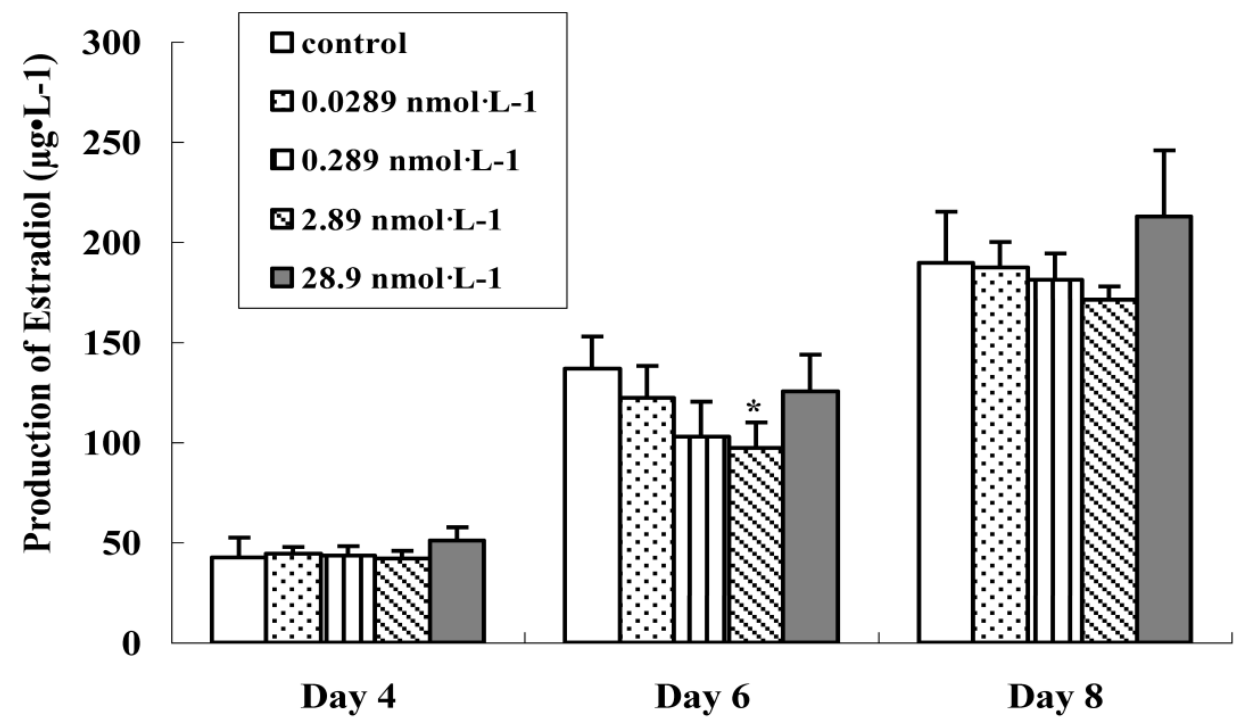

Figure 6. Effect of QDs-Tf for production of $17 \beta$-estradiol on the $4^{\text {th }}, 6^{\text {th }}$ and $8^{\text {th }}$ days. (mean $\pm S D, * P<0.05, A N O V A$ test) 


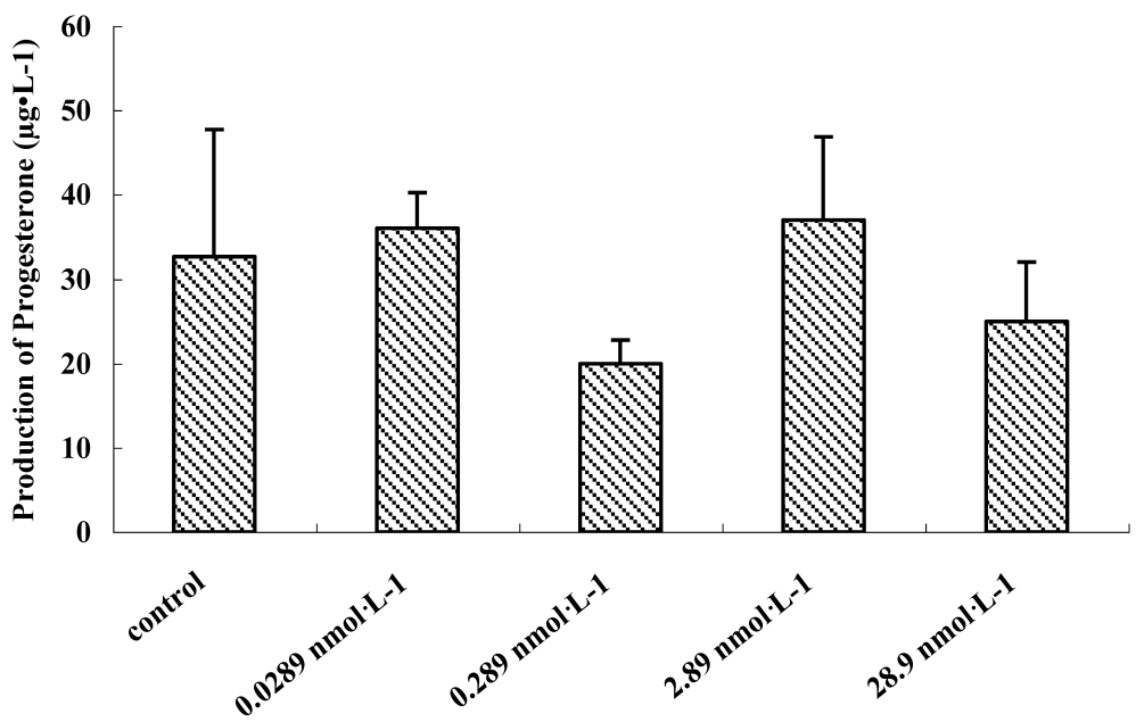

Figure 7. Effect of QDs-Tf for production of progesterone on the 9th day (mean $\pm S D, A N O V A$ test)

\section{Effect of QDs-Tf on oocyte cytoplasmic mat- uration}

To evaluate the effect of QDs-Tf on the oocyte cytoplasmic maturation, we calculated the ratio of oocyte at different phases. It has been known that GV oocytes are maturation incompetent, GVBD oocytes contains distinguishable nuclear membrane and one set of chromosomes which does not emit the first polar body, and PB oocytes have two sets of chromosomes and the cytokinesis process can be easily detected. After being stimulated by HCG and EGF, COCs mucified, the denuded oocytes were transferred individually into another dish droplets and assigned into 3 groups (i.e. GV oocytes, GVBD oocytes and PB oocytes). The results showed that the denuded oocyte diameters between the treated groups and control group had no significant difference $(n=95, \quad P>0.05)$. At lower QDs-Tf concentraion, the percentages of PB oocytes, GVBD oocytes and GV oocytes were similar for all the experimental groups (Figure 8). However, the PB oocyte decreased significantly from $79.6 \pm 10 \%$ to 63 $\pm 2.9 \%$ when the concentration of QDs-Tf is higher than $2.89 \mathrm{nmol} \cdot \mathrm{L}^{-1} \quad(\mathrm{P}<0.05)$. The results confirmed that higher concentration of QDs-Tf could delay the antrum formation and decrease the ratio of $\mathrm{PB}$ oocyte dramatically.

\section{Effect of QDs-Tf on oocyte nuclear maturation}

To explore the effect of QDs-Tf on the nuclear maturation of oocyte, we analyzed the chromosome configuration and numbers, respectively. Firstly, the chromosome configuration at different oocyte meiotic stage were observed and counted. GVBD oocytes possessed bivalents (MI, Figure 9A), or two sets of dyads (MI: anaphase I, Figure 9B). Then, oocytes emitted the first polar body and entered into metaphase II (Figure 9C). PB oocytes contained either one set of dyads according to metaphase II stage or two sets of chromatids when they became spontaneously activated and progressed to anaphase II during culture. We also observed the split of chromatids (Figure 9D).

The statistical data was shown in Table 3. Although, the ratios of oocyte at bivalent of meiosis I treated with $28.9 \mathrm{nmol} \cdot \mathrm{L}^{-1}$ QDs-Tf (MI:Biv, Figure 9A), at anaphase I of meiosis I treated with 2.89 $\mathrm{nmol} \cdot \mathrm{L}^{-1}$ QDs-Tf (MI:Anaph I , Figure 9B), and at anaphase II of meiosis II treated with $2.89 \mathrm{nmol} \cdot \mathrm{L}^{-1}(\mathrm{M}$ II :Anaph II , Figure 9D), were twice than those of control groups, respectively, the ratio of oocyte nucleus at metaphase of meiosis II did not differ significantly for all experimental groups $(\mathrm{P}>0.05)$.

Table 3 showed that the chromosome configuration had not been affected by the QDs-Tf within the concentration used in this work.

Beside the chromosome configuration, the chromosomal numerical abnormalities were also investigated. The percentage of hypoploidy $(n<20$, Figure 10A) and hyperploidy ( $>>20$, Figure 10B) were statistically analyzed under a microscope Table 4 showed that small amount of pre-divisions of chromosome (as illustrated in Figure 9D) were found in almost all treated groups except at the concentration of $0.289 \mathrm{nmol} \mathrm{L}^{-1}$. However, such small variations had no significant difference $(\mathrm{P}>0.05)$ to the control group. 
The results demonstrated that within the QDs-Tf concentrations used in this work, minimal oocyte chromosomal abnormalities including configuration and numerical aberrations were observed, suggesting that the oocyte nuclear maturation was not affected by the QDs-Tf.

Table 3. Effect of QDs-Tf on Chromosome configuration.

\begin{tabular}{|c|c|c|c|c|c|}
\hline Groups & $\mathrm{N}$ & M I :Biv(\%) & M I :Anaph I (\%) & M II :Meta II (\%) & M II :Anaph II (\%) \\
\hline Control & 87 & $2(2.3)$ & $2(2.3)$ & $81(93.1)$ & $2(2.3)$ \\
\hline $0.0289 \mathrm{nmol} \cdot \mathrm{L}^{-1}$ & 73 & $2(2.7)$ & $3(4.1)$ & $66(90.4)$ & $2(2.7)$ \\
\hline $0.289 \mathrm{nmol} \cdot \mathrm{L}^{-1}$ & 62 & $1(1.6)$ & $2(3.2)$ & $59(95.2)$ & $0(0)$ \\
\hline $2.89 \mathrm{nmol} \mathrm{L}^{-1}$ & 78 & $2(2.6)$ & $4(5.2)$ & $67(85.9)$ & $5(6.4)$ \\
\hline $28.9 \mathrm{nmol} \cdot \mathrm{L}^{-1}$ & 65 & $3(4.6)$ & $1(1.5)$ & $59(90.8)$ & $2(3.1)$ \\
\hline
\end{tabular}

(N, number of oocytes, vs control group).

Table 4. Effect of QDs-Tf on chromosome number.

\begin{tabular}{llllll}
\hline Groups & $\mathrm{N}$ & Diploid(\%) & Hypoploid(\%) & Hyperploid(\%) & Pre-division(\%) \\
\hline Control & 83 & $72(86.8)$ & $8(9.6)$ & $1(1.2)$ & $2(2.4)$ \\
$0.0289 \mathrm{nmol} \cdot \mathrm{L}^{-1}$ & 68 & $60(88.3)$ & $6(8.8)$ & 0 & $2(2.9)$ \\
$0.289 \mathrm{nmol} \cdot \mathrm{L}^{-1}$ & 59 & $53(89.8)$ & $6(10.2)$ & 0 & 0 \\
$2.89 \mathrm{nmol} \mathrm{L}-1$ & 72 & $59(82.0)$ & $5(9.7)$ & $1(1.4)$ & $5(6.9)$ \\
$28.9 \mathrm{nmol} \mathrm{L}^{-1}$ & 61 & $54(88.5)$ & & 0 & $2(3.3)$ \\
\hline
\end{tabular}

(N, number of oocytes, vs control group)

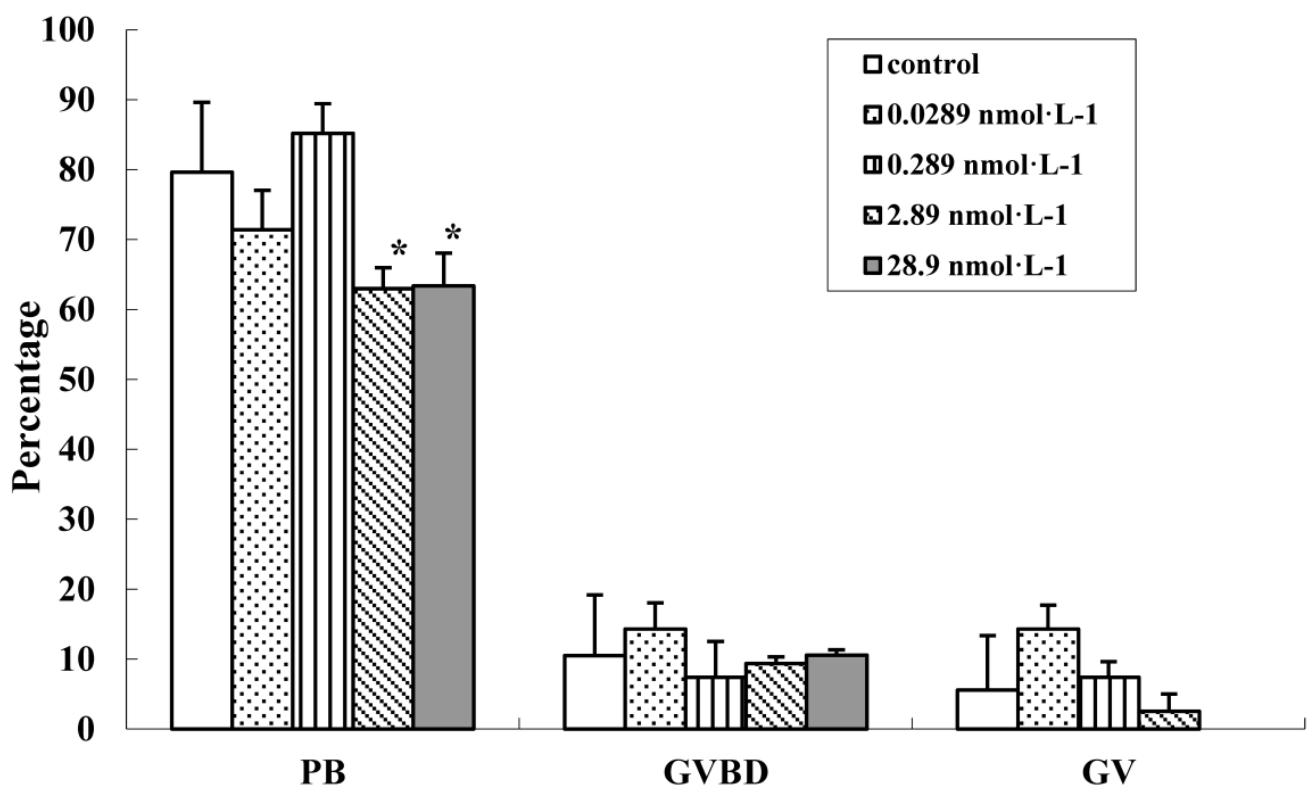

Figure 8 Comparision of cytoplasmic maturation of oocytes (mean $\pm S D, * P<0.05$ vs control, Chi-Square test). 

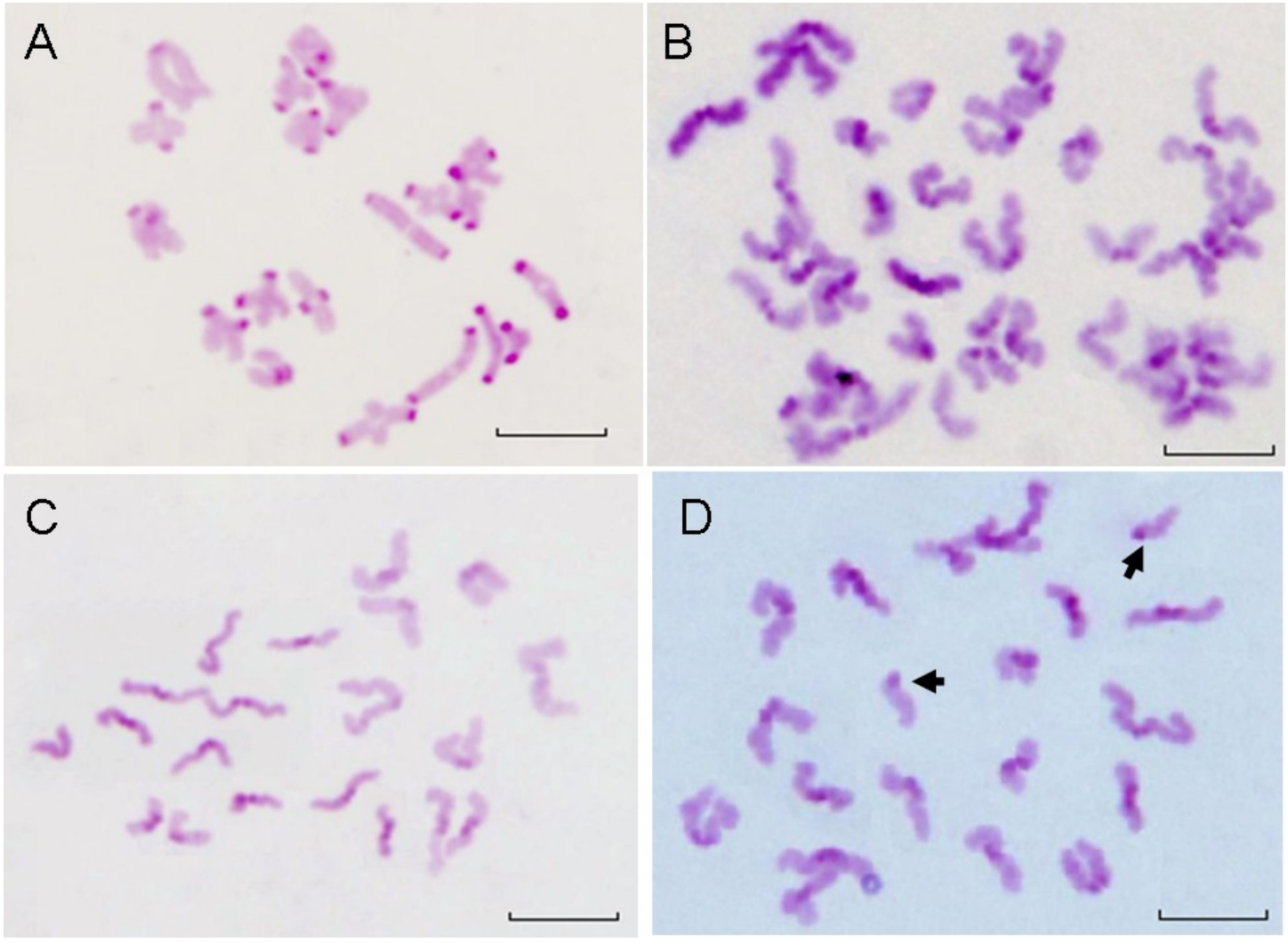

Figure 9. The chromosomes of GVBD and PB oocytes treated with QDs-Tf $\left(2.89 \mathrm{nmol} \mathrm{L}^{-1}\right)$. (A) Bivalent chromosomes in meiosis I oocyte. (B) Chromosomes in anaphase I of meiosis, with two sets of dyads. (C) Normal Chromosomes in metaphase II with one set of dyads. (D) Hyperploid oocyte with 19 dyads and 2 single chromatids (arrows). Scale bar: $10 \mu \mathrm{m}$.
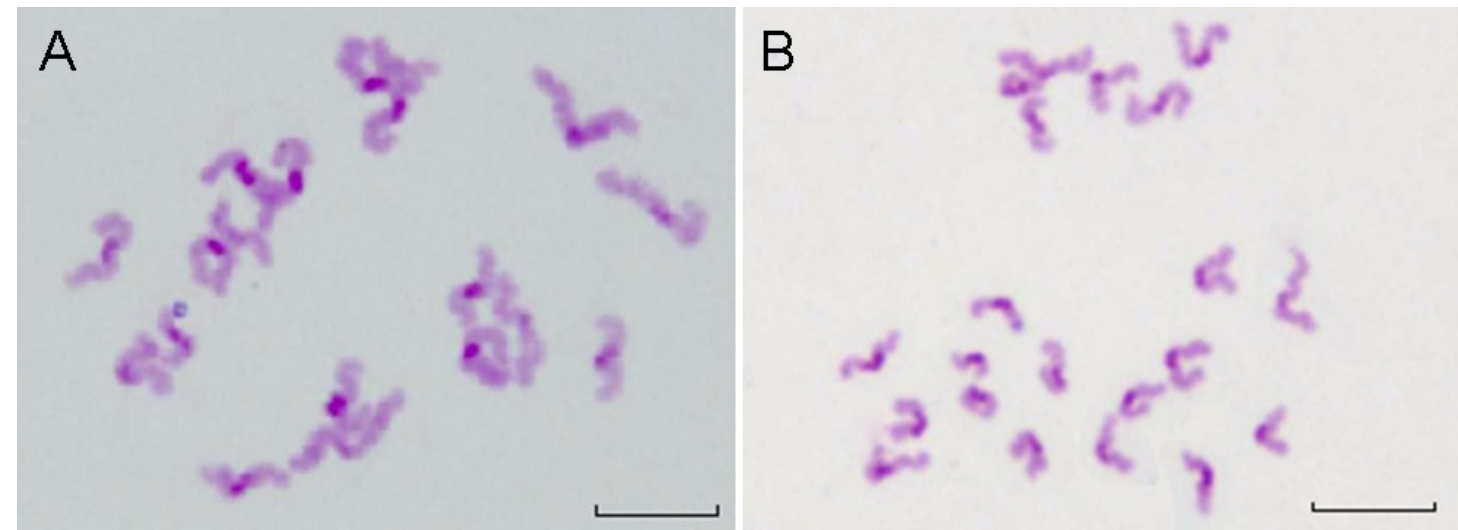

Figure 10. The chromosomes of PB oocytes treated with QDs-Tf (2.89 nmol $\left.\mathrm{L}^{-1}\right)$. (A) Hypoploid with 19 dyads in oocyte. (B) Hyperploid with 21 dyds in oocyte. Scale bar: $10 \mu \mathrm{m}$.

\section{Conclusion}

In this study, the preantral follicles were cultured and exposed to CdTe/ZnTe QDs-Tf bioconjugates with various concentrations, and the reproductive toxicity was assessed at different time point post-treatment. We found that the preantral follicles survived for more than 8 days at all of the QDs-Tf bioconjugate dosage and the follicle-enclosed oocytes resumed their maturation and developed into metaphase II after the addition of HCG and EGF. We also found that most of the QDs-Tf bioconjugates were uptaken by granulosa cells and theca cells, and then the excess amount of bioconjugates was blocked by 
the zona pellucida surrounding the oocyte. The oocyte diameter, the mucification of COCs and the ratios of metaphase I oocytes and metaphase II oocytes has no significant differences $(p>0.05)$ as compared with the control group. However, the maturity rate of oocytes with first polar body was affected and the oocyte antrum cavity formation was delayed after the treatment of QDs-Tf bioconjugates. These results implied that the CdTe/ZnTe QDs-Tf bioconjugates disturbed the oocyte cytoplasmic maturation but had no effect on the nuclear maturation. This work demonstrated that the preantral follicle in vitro growth system is powerful to assess the alterations in the microenvironment of the follicle and oocyte, which provided a highly sensitive platform to explore the reproductive effect and toxicological mechanism of nanomaterials.

\section{Acknowledgments}

We gratefully acknowledge Professor Qingning $\mathrm{Su}$ for her valuable comments and suggestions on the revised manuscript. This research was supported by National Natural Science Foundation of China (NO. 30900335), National Basic Research Program of China (NO. 2012CB85802), Scientific Research Starting Foundation for Returned Overseas Chinese Scholars, Ministry of Education, China, Natural Science Foundation of Guangdong Province (NO.2008078 and NO. S2011010001223) and Basic Research of Shenzhen (JC201005280500A).

\section{Competing Interests}

The authors have declared that no competing interest exists.

\section{References}

1. Alivisatos $\mathrm{AP}, \mathrm{Gu}$ WW, Larabell C. Quantum dots as cellular probes. Annual Review of Biomedical Engineering. 2005;7:55-76.

2. Gao XH, Yang LL, Petros JA, Marshal FF, Simons JW, Nie SM. In vivo molecular and cellular imaging with quantum dots. Current Opinion in Biotechnology. 2005;16:63-72

3. Ye YP and Chen XY. Integrin targeting for tumor optical imaging. Theranostics. 2011;1:102-126.

4. Chan WCW, Nie SM. Quantum dot bioconjugates for ultrasensitive nonisotopic detection. Science. 1998;281:2016-2018.

5. Michalet X, Pinaud F F, Bentolila LA, Tsay JM, Doose S, Li JJ, et al. Quantum dots for live cells, in vivo imaging, and diagnostics. Science. 2005;307:538-544.

6. Oberdorster G, Oberdorster E, Oberdorster J. Nanotoxicology: An emerging discipline evolving from studies of ultrafine particles. Environmental Health Perspectives. 2005;113:823-839.

7. Taylor U, Barchanski A, Garrels W, Klein S, Kues W, Barcikowski S, et al. Toxicity of gold nanoparticles on somatic and reproductive cells. Advances in Experimental Medicine and Biology. 2012;733:125-133.

8. Kirchner C, Liedl T, Kudera S, Pellegrino T, Javier AM, Gaub HE, et al. Cytotoxicity of colloidal CdSe and CdSe/ZnS nanoparticles. Nano Letters. 2005;5:331-338.

9. Hardman R. A Toxicologic Review of Quantum Dots: Toxicity Depends on Physicochemical and Environmental Factors. Environmental Health Perspectives. 2006;114:165-172.
10. Li KG, Chen JT, Bai SS, Wen X, Song SY, Yu Q, et al. Intracellular oxidative stress and cadmium ions release induce cytotoxicity of unmodified cadmium sulfide quantum dots. Toxicology In Vitro. 2009;23:1007-1013.

11. Cortvrindt RG, Smitz J. Follicle culture in reproductive toxicology: a tool for in-vitro. Human Reproduction Update. 2002;8:243-254.

12. Fynewever TL, Agcaoili ES, Jacobson JD, Patton WC, Chan PJ. In vitro tagging of embryos with nanoparticles. Journal of Assisted Reproduction and Genetics. 2007;24:61-65.

13. Sun FY, Betzendahl I, Shen Y, Cortvrindt R, Smitz J, Eichenlaub-Ritter U. Preantral follicle culture as a novel in vitro assay in reproductive toxicology testing in mammalian oocytes. Mutagenesis. 2004;19:13-25.

14. Eppig JJ, Schroeder AC. Capacity of mouse oocytes from preantral follicles to undergo embryogenesis and development to live young after growth, maturation, and fertilization in vitro. Biology of reproduction. 1989;41:268-276.

15. Nayudu PL, Kiesel PS, Nowshari MA, Hodges JK. Abnormal in vitro development of ovarian follicles explanted from mice exposed to tetrachlorvinphos. Reproductive Toxicology. 1994;8:261-268.

16. Lin $\mathrm{XT}, \mathrm{Xu}$ GX, Yong KT, Roy I, Qu JL, Wang XM. Visualization of reproduction toxicity of QDs for in vitro oocytes maturation. Proceedings of SPIE. 2009;7519: 75191W.

17. Qian J, Yong KT, Roy I, Ohulchanskyy TY, Bergey EJ, Lee HH, et al. Imaging pancreatic cancer using surface functionalized quantum dots. The Journal of Physical Chemistry B. 2007;111:6969-6972.

18. Gerard VA, Maguire CM, Bazou D, Gunko Y K. Folic acid modified gelatine coated quantum dots as potential reagents for in vitro cancer diagnostics. Nanobiotechnology. 2011;9:50.

19. Lécureuil C, Staub C, Fouchécourt S, Maurel MC, Fontaine I, Martinat N, et al. Transferrin overexpression alters testicular function in aged mice. Molecular Reproduction and Devvelopment. 2007;74:197-206.

20. Law WC, Yong KT, Roy I, Ding H, Hu R, Zhao WW, et al. Aqueous-Phase Synthesis of Highly Luminescent CdTe/ZnTe Core/Shell Quantum Dots Optimized for Targeted Bioimaging. Small. 2009;5:1302-1310.

21. Liu X, Chen DN, Qu JL, Yang JT, Luo YX, Roy I, et al. Characterization of biocompatible CdTe/ZnTe quantum dots and its application in cell labelling. Spectroscopy and Spectral Analysis. 2010;30:1290-1294.

22. Cortvrindt R, Smitz J, Van Steirteghem AC. In-vitro maturation, fertilization and embryo development of immature oocytes from early preantral follicles from prepuberal mice in a simplified culture system. Human Reproduction. 1996;11:2656-2666.

23. Sun F, Yin H, Eichenlaub-Ritter U. Differential chromosome behavior in mammalian oocytes exposed to the tranquilizer diazepam in vitro. $\mathrm{Mu}-$ tagenesis. 2001;16:407-417.

24. Eichenlaub-Ritter U, Boll I. Nocodazole sensitivity, age-related aneuploidy, and alterations in the cell cycle during maturation of mouse oocytes. Cytogenetics and Cell Genetics. 1989;52:170-176.

25. Jang JS, Javidi B. Improved viewing resolution of three- dimensional imaging by use of nonstationary micro-optics. Optics Letters. 2002;27:324-326.

26. Stelzer R, Hutz RJ. Gold nanoparticles enter rat ovarian granulosa cells and subcellular organelles, and alter in-vitro estrogen accumulation. Jouranl of Reproduction and Development. 2009;55:685-690.

27. Wang XM, Yong KT, Xu GX, Lin XT, Zhou XQ, Qu JL, et al. Invasion of CdSe/CdS/ZnS Quantum Dots for Oocytes in Vitro Maturation. Chinese Laser. 2010;37:2730-2734.

28. Cortvrindt R, Smitz J, Van Steirteghem AC. Assessment of the need for follicle stimulating hormone in early preantral mouse follicle culture in vitro. Human Reproduction. 1997;12:759-768.

29. Cortvrindt R, Hu Y, Liu J, Smitz J. Timed analysis of the nuclear maturation of oocytes in early preantral mouse follicle culture supplemented with recombinant gonadotropin. Fertililty and Sterility. 1998;70:1114-1125. 